\title{
Coherent Diabatic Ion Transport and Separation in a Multizone Trap Array
}

\author{
R. Bowler, ${ }^{*}$ J. Gaebler, Y. Lin, T. R. Tan, D. Hanneke, ${ }^{\dagger}$ J. D. Jost, J. P. Home, ${ }^{\ddagger}$ D. Leibfried, and D. J. Wineland \\ National Institute of Standards and Technology, 325 Broadway, Boulder, Colorado 80305
}

(Received 4 June 2012; published 20 August 2012)

\begin{abstract}
We investigate the dynamics of single and multiple ions during transport between and separation into spatially distinct locations in a multizone linear Paul trap. A single ${ }^{9} \mathrm{Be}^{+}$ion in a $\sim 2 \mathrm{MHz}$ harmonic well was transported $370 \mu \mathrm{m}$ in $8 \mu \mathrm{s}$, corresponding to 16 periods of oscillation, with a gain of 0.1 motional quanta. Similar results were achieved for the transport of two ions. We also separated chains of up to 9 ions from one potential well to two distinct potential wells. With two ions this was accomplished in $55 \mu \mathrm{s}$, with excitations of approximately two quanta for each ion. Fast transport and separation can significantly reduce the time overhead in certain architectures for scalable quantum information processing with trapped ions.
\end{abstract}

DOI: 10.1103/PhysRevLett.109.080502

For quantum information processing based on trapped ions [1], scaling might be achieved with an array of interconnected trap zones where information is transported by confining the ions in potential wells that are moved between zones [2,3]. The basic features of this scheme have been demonstrated with adiabtic transport of ion qubits on a time scale much greater than the period of oscillation of ions in their local wells. This suppresses motional excitation that can impede the ability to perform high-fidelity multiqubit quantum logic gates [4-8]. In [9], one and two ions were separated from a group of three in $2 \mathrm{~ms}$ with a mode excitation of less than one quantum. In [5-7], a mixed-ion linear chain or "crystal" of ions was separated into two ${ }^{9} \mathrm{Be}^{+}{ }^{24} \mathrm{Mg}^{+}$pairs accompanied by motional excitation that could be removed with sympathetic laser cooling; however, the time required for ion separation, transport, and sympathetic laser cooling was 100 times larger than for logic gates, emphasizing the need for improvement.

To reduce this time overhead we have investigated diabatic transport and separation. Previously, diabatic ion transport was accomplished in only four oscillation periods, but this resulted in large motional excitation [10]. Diabatic transport with small final excitation of the center-of-mass (c.m.) motion has been observed for cold neutral-atom ensembles on $1 \mathrm{~s}$ time scales (approximately a single oscillation period) [11].

Transport and separation of ions can be implemented by applying time-varying potentials (waveforms) to segmented trap electrodes. In our experiments, ions are confined in a multizone linear Paul trap (Fig. 1) in which an ion's local potential well can be made approximately harmonic. To determine the overall potential at any given time, we use simulations of potentials from individual electrodes that are linearly superposed. Along the trap axis (horizontal in Fig. 1), the confinement is characterized by (angular) frequency $\omega$. Confinement in the transverse direction is significantly stronger and does not play a
PACS numbers: 03.67.Lx, 37.10.De, 37.10.Gh, 37.10.Ty

significant role here. Waveform potentials are updated at $50 \mathrm{MHz}$, enabling considerable changes in potentials within single periods of oscillation. Zones $A, X$, and $B$ are labeled in Fig. 1.

The qubit is formed from two $2 \mathrm{~s}{ }^{2} \mathrm{~S}_{1 / 2}$ electronic ground-state hyperfine levels of ${ }^{9} \mathrm{Be}^{+}\left|F=2, m_{F}=1\right\rangle \equiv|\downarrow\rangle$ and $\left|F=1, m_{F}=0\right\rangle \equiv|\uparrow\rangle$, separated in frequency by $\omega_{0} /(2 \pi) \simeq 1.2 \mathrm{GHz}$, where $F$ and $m_{F}$ are the ion's total angular momemtum and its projection along the quantizing magnetic field $(\simeq 11.9 \mathrm{mT})$. Ion motional quantum states can be described in the Fock state basis $|n\rangle$ with energy $\hbar \omega\left(n+\frac{1}{2}\right)$. To characterize the motional states experimentally, we use two laser beams detuned from each other by $\omega_{0}+\omega$ or $\omega_{0}-\omega$ to drive motion adding sideband (MAS) spin-flip transitions $|\downarrow, n\rangle \rightarrow|\uparrow, n+1\rangle$ or motion subtracting sideband (MSS) transitions $|\downarrow, n\rangle \rightarrow|\uparrow, n-1\rangle$, respectively, via stimulated-Raman transitions. Since the corresponding Rabi rates $\Omega_{n, n \pm 1}$ are $n$ dependent, the populations $P_{n}$ of Fock states can be determined from the probability $P_{\downarrow}(\mathrm{t})$ of the state $|\downarrow\rangle$ as a function of sideband drive time $t$, derived from state-dependent resonance fluorescence [12]. As an approximation, we fit to

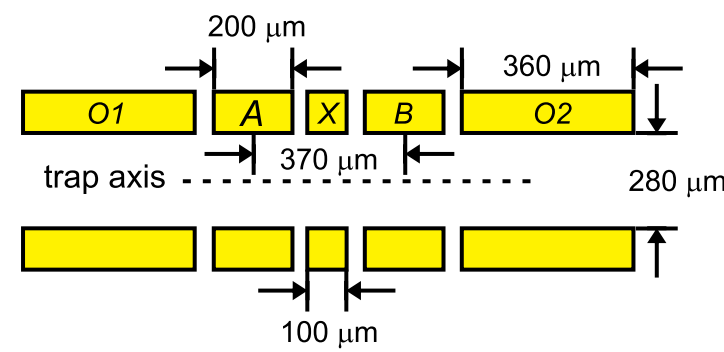

FIG. 1 (color online). Schematic of the linear Paul (quadrupole) ion trap electrode structure showing the two diagonally opposite segmented dc electrodes (not to scale; trap details in [5-7,21]). Ions are loaded in a region to the left of zone $O 1$ and initially transported to zone $A$. Trap radio-frequency (rf) electrodes (not shown) are referenced to a common ground potential. 
the function $P_{\downarrow}(t)=\frac{1}{2}\left[1+e^{-\gamma t} \sum_{n=0} P_{n} \cos \left(2 \Omega_{n, n \pm 1} \mathrm{t}\right)\right]$ to include a phenomenological decay rate $\gamma$. Acousto-optic deflectors directed the laser beams to either zone $A$ or $B$.

There have been several theoretical investigations on diabatic transport with minimally excited final states [10,13-16]. In our experiments, waveforms are designed to keep $\omega$ constant so that in the frame of the well, the ion motion is in a coherent state [17] characterized by a complex amplitude $\alpha$ where $|\alpha|^{2}=\bar{n}$. For transport along $z$ we can write the potential as $\frac{1}{2} m \omega^{2}\left[z-z_{0}(t)\right]^{2}$, where $m$ is the mass of the ion and $z_{0}(t)$ is the minimum of the well. From [16], the final state after transport is a coherent state with

$$
\alpha(t)=\sqrt{\frac{m \omega}{2 \hbar}}\left[-e^{-i \omega t} \int_{0}^{t} \dot{z}_{0}\left(t^{\prime}\right) e^{i \omega t^{\prime}} d t^{\prime}\right] .
$$

As long as the Fourier component of $\dot{z}_{0}\left(t^{\prime}\right)$ at the trap frequency vanishes when integrated over the transport duration, the ion will end up in its initial state at position $z_{0}(t)$. When the well impulsively starts moving at constant velocity $v$ and then stops at time $t_{T}\left(\dot{z}_{0}=0\right.$ for $t<0$ and $t>t_{T}$, and $\dot{z}_{0}=v$ for $t \in\left[0, t_{T}\right]$ ),

$$
\alpha\left(t_{T}\right)=\sqrt{\frac{m \omega}{2 \hbar}}\left(i \frac{v}{\omega}\left[1-e^{-i \omega t_{T}}\right]\right) .
$$

For $\omega t_{T}=2 \pi N$, with $N$ an integer, an ion starting in its ground state of motion is caught back in the ground state, as in [11]. Because of the finite response times of our electrode drive electronics, sudden acceleration is not possible but Eq. (1) indicates that one can always find a coherent displacement that returns the ion to its ground-state.

As a demonstration of diabatic transport, a single ion in zone $A\left[\omega /(2 \pi) \simeq 2 \mathrm{MHz} ;\left\{V_{O 1}, V_{A}, V_{X}, V_{B}, V_{O 2}\right\}=\right.$ $\{1.289,0.327,2.173,0.310,1.311\} V]$ was first initialized; it was laser cooled to a thermal distribution with $\bar{n} \approx 0.1$ and optically pumped to $|\downarrow\rangle$. It was then transported $370 \mu \mathrm{m}$ in $t_{T} \approx 8 \mu$ s to zone $B(A \rightarrow B)$ with a waveform that approximated the conditions of Eq. (2). Following transport, qubit sideband transitions were driven in zone $B$, and then the ion was transported back to zone $A(B \rightarrow A)$ to determine $P_{\downarrow}(t)$. By varying $\omega$, we found a minimal final excitation of $\bar{n}=0.19 \pm 0.02$ from the MAS fit and $\bar{n}=$ $0.17 \pm 0.01$ from the MSS fit (Fig. 2). The axial frequency was $\omega /(2 \pi)=1.972(1) \mathrm{MHz}$, corresponding approximately to $N=16$. Excitation was also minimized for values of $\omega / 2 \pi$ differing from this value by integer multiples of $t_{T}^{-1}$.

For other values of $\omega /(2 \pi), P_{\downarrow}(t)$ measurements were consistent with coherent state probability distributions over Fock states; however, these measurements do not verify the coherences between Fock states. To verify coherence, we implemented $A \rightarrow B$ transport in a well of frequency $\omega /(2 \pi)=1.919(2) \mathrm{MHz}$ such that $\alpha\left(t_{T}\right) \neq 0$ in zone $B$ (Fig. 3). We then applied a uniform electric field $E_{z}=$ $E_{0} \cos \left(\omega t_{E}+\phi_{E}\right)$ to provide a phase-space displacement $\alpha_{E}$. This was followed by a sideband drive of duration $t$,

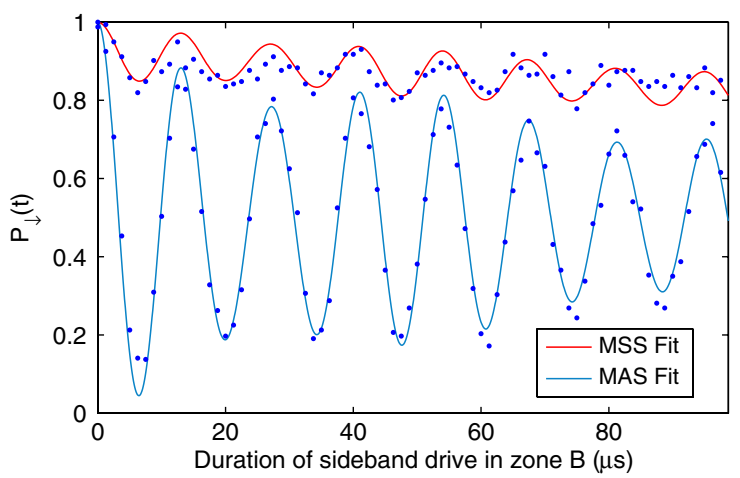

FIG. 2 (color online). Rabi flopping trace on the MAS and MSS after transport from zone $A$ to $B$ [12]. Here we fit to thermal distributions that give $\bar{n}=0.19 \pm 0.02$ from the MAS fit and $\bar{n}=0.17 \pm 0.01$ from the MSS fit. The axial frequency was $\omega /(2 \pi)=1.972(1) \mathrm{MHz}$ with a Lamb-Dicke parameter $\eta=0.479$.

transport back to zone $A$, and measurement of $P_{\downarrow}(t)$. By adjusting $E_{0}, t_{E}$, and $\phi_{E}$ (relative to the time when transport started), we could make $\alpha_{E}=-\alpha\left(t_{T}\right)$. Under these conditions, we measured a final state at $\bar{n}=0.19 \pm 0.02$. In addition, when $\alpha\left(t_{T}\right) \neq 0$ in zone $B$, by waiting an appropriate delay $T_{d} \pm N 2 \pi / \omega$ in $B$, and then transporting back to zone $A$, the ion would be returned to its ground state in $A$ ( $A \rightarrow B \rightarrow A$, "cold" round-trip transport).

Internal state qubit coherences are maintained during transport $[4,8]$. Under transport with minimal final excitation any Fock state receives the same phase-space displacement across the transport; therefore, initial motional states should maintain the same relative coherences before and after transport. We demonstrate this in a Ramsey-type interference experiment. After state initialization, a MAS $\pi / 2$ pulse transformed the state to $\frac{1}{\sqrt{2}}(|\downarrow, n=0\rangle+\mid \uparrow$, $n=1\rangle$ ). We then transported the ion $A \rightarrow B \rightarrow A$ with the motional state close to the ground state after each leg. A second MAS $\pi / 2$ pulse of variable phase produced

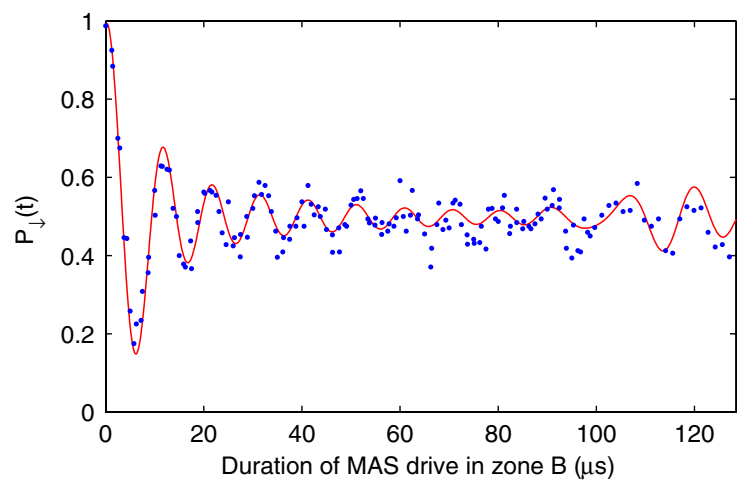

FIG. 3 (color online). Rabi flopping trace on the MAS following transport in $8 \mu \mathrm{s}$ in which $\alpha\left(t_{T}\right) \neq 0$. The figure shows a coherent state fit with $\bar{n}=6.4 \pm 0.2$, corresponding to $|\alpha|=$ $2.53 \pm 0.04$. The axial frequency was $\omega /(2 \pi)=1.919(2) \mathrm{MHz}$ and the Lamb-Dicke parameter was $\eta=0.486$. 
Ramsey fringes of $86(2) \%$ contrast, limited primarily by imperfect initial ground-state cooling and the relatively large Lamb-Dicke parameter. For reference, we performed a Ramsey experiment with free precession time equal to the transport duration, but the ion held stationary in zone $A$. We measured a fringe contrast of $85(2) \%$, consistent with no loss in coherence due to transport.

We also transported two ${ }^{9} \mathrm{Be}^{+}$ions $(A \rightarrow B)$ in the same well. If the moving potential well is maintained at constant frequency, the "stretch" mode (ion motion out of phase) should not be excited and the c.m. mode should be excited as for a single ion. We initialized both modes to $\bar{n} \approx 0.1$ and after cold transport observed $\bar{n}_{\text {c.m. }}=0.35 \pm 0.1$, and negligible additional excitation of the stretch mode.

When separating multiple ions confined in a single well into two separate wells, it is impossible to preserve the motional mode frequencies. To lowest order, the required external potential can be described as the sum of a quadratic and a quartic term for $0 \leq t \leq t_{s}$ during separation [18]:

$$
U(z)=a(t) z^{2}+b(t) z^{4},
$$

with $a(0)>0, b(0)=0$ and $a\left(t_{s}\right)<0, b\left(t_{s}\right)>0$. Because of Coulomb repulsion, the normal modes of multiple ions remain harmonic throughout the separation for small excursions about each ion's local minimum [18]. The normalmode frequencies go through their minima near the point when $a$ vanishes and the quartic potential dominates the confinement.

Our separation waveform consisted of two main segments. Ions were first transported to zone $X$, whose center is defined as $z=0$ relative to Eq. (3). Then $a(t)$ was decreased and $b(t)$ increased by lowering the potentials on electrodes $A$ and $B$ while simultaneously increasing the potentials on outer electrodes $O 1$ and $O 2$ until the mode frequencies approximately reach their minimum values. In the second segment, we separated the ions into distinct wells in zones $A$ and $B$ by increasing the potential on electrode $X$ such that $a(t)$ changed sign, creating a "wedge" that splits the ions apart.

As one demonstration of ion separation control, we ran a separation waveform (duration $\approx 340 \mu \mathrm{s}$ ) to partition a linear crystal of nine ${ }^{9} \mathrm{Be}^{+}$ions into all possible combinations. We first Doppler cooled the crystal in zone $A$ and optically pumped to $\left|\downarrow_{1}, \downarrow_{2}, \ldots, \downarrow_{9}\right\rangle$, the fluorescing state. It was then transported to zone $X$ for separation into two groups in zones $A$ and $B$. A variable offset potential $V_{O 2}$ applied to electrode $O 2$ imposed an electric field in zone $X$ that shifted the center of the crystal relative to the separation wedge and determined the number of ions in each group. Ions in zone $A$ were detected with laser-induced fluorescence, approximately proportional to ion number. Following detection, all ions were recombined and transported to zone $A$ where the number was checked. As shown in Fig. 4, increasing $V_{O 2}$ led to increased ion numbers in zone $A$. By adjusting $V_{O 2}$ to the center of a certain step, we

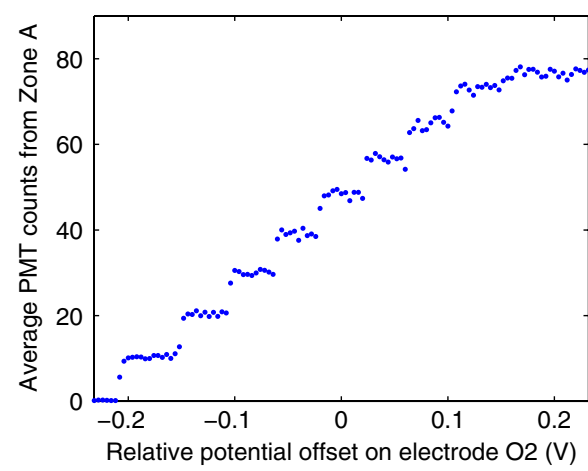

FIG. 4 (color online). Ion fluorescence counts in zone $A$ as a function of the offset potential $V_{O 2}$ during separation relative to a central value. Each step increase of the counts corresponds to the presence of an additional ion in zone $A$ after separation. A single ion produced approximately 10 average photomultiplier tube (PMT) counts in $200 \mu \mathrm{s}$, but as more ions were added in zone $A$, the crystal was no longer uniformly illuminated by the detection beam and the signal dropped below 10 counts per ion.

could reliably partition the ions into two groups of a predetermined number.

During separation, diabatic changes in both curvature and minimum position of the axial potential can lead to coherent displacement and squeezing of the motional states. These excitations can be suppressed by approximating the adiabatic condition for ramping a harmonic well, $\frac{1}{\omega^{2}} \frac{d \omega}{d t} \ll 1$ [19]. For two-ion separation, we numerically solved for this quantity in our waveform and imposed a maximum value of $\frac{1}{\omega^{2}} \frac{d \omega}{d t}=0.025$ for the c.m. mode during the ramp-down of the harmonic well and $\frac{1}{\omega^{2}} \frac{d \omega}{d t}=0.015$ around the sign change of $a$ in Eq. (3).

A two-ion crystal in zone $X$ with an initial well frequency of $\omega /(2 \pi)=2.6 \mathrm{MHz}\left(\left\{V_{O 1}, V_{A}, V_{X}, V_{B}, V_{O 2}\right\}=\right.$ $\{2.433,-0.3763,-1.7089,-0.3831,2.473\} V)$ could be separated and one ion placed in zones $A$ and $B$ (with motional frequencies 2.85 and $2.77 \mathrm{MHz}$, respectively, $\left\{V_{O 1}, \ldots, V_{O 2}\right\}=\{4.441,-5.252,-0.649,-5.411$, $5.952\} \mathrm{V}$ ) in a duration of $55 \mu \mathrm{s}$. After separation, qubit MAS transitions of varying duration $t$ were driven on either the ion in zones $A$ or $B$ followed by recombination in zone $X$, whereupon fluorescence state detection was performed to determine $P_{\downarrow}(t)$ for each ion. The first separation segment took $17 \mu \mathrm{s}$, during which it was necessary to ramp $V_{O 2}$ from 2.473 to $5.952 \mathrm{~V}$ to compensate for trap geometric asymmetries and to center the crystal over the separation wedge; the final value was set by minimizing $\bar{n}$ for both separated ions. Near the point of ion separation, the minimum c.m. mode frequency was $700 \mathrm{kHz}$ and minimum stretch mode frequency was $880 \mathrm{kHz}$. During the second separation segment we obtained best results by also applying a variable offset potential to electrode $X$ to tune the quartic component $b(t)$ in the potential. We also applied a relative difference between electrodes $A$ and $B$ to compensate for trap imperfections and make the potential 
well as symmetric as possible with respect to electrode $X$. This enabled a fine-tuned balancing of the final excitation between the two ions. Experimentally, we found that equal excitation in both final zones gave the lowest total excitation to both ions.

The two-ion crystal was initialized in zone $X$ to a thermal state with $\bar{n} \approx 0.1$ on both the c.m. and stretch modes and optical pumping to $\left|\downarrow_{1} \downarrow_{2}\right\rangle$. After separation, sideband excitation gave Fock state populations consistent with coherent states of $\bar{n}=2.1 \pm 0.1$ in zone $A$ and $\bar{n}=$ $1.9 \pm 0.1$ in zone $B$. With rf deexcitation of each ion, we could reduce $\bar{n}$ to $1.4 \pm 0.1$ and $1.6 \pm 0.1$ in zones $A$ and $B$, respectively, which is consistent with a thermal distribution. The reductions indicated partial coherence of the final states. The fact that we could not reduce $\bar{n}$ further might be explained from the sensitivity of final conditions to (fluctuating) potentials near separation. For example, for a change of $\mathrm{V}_{O_{2}}$ of $+3 \mathrm{mV}$, the motion of the ion in zone $A$ had $\bar{n} \simeq 1.14 \pm 0.07$, while the ion in zone $B$ had $\bar{n}>15$, which then could be coherently deexcited to a state consistent with a thermal distribution having $\bar{n}=3.8 \pm 0.5$. The large excitation of the ion in $B$ can be qualitatively explained; since it is closer to the maximum of the wedge during separation, it would be expected to gain more kinetic energy as it accelerates towards the center of its respective well. Simulations of ambient heating using measured values of heating vs $\omega$ for a single ion predicted only $\Delta \bar{n} \approx 0.2$.

In summary, we have demonstrated diabatic transport of one and two ions and the separation of two ions in a multizone linear trap on $10 \mu$ s time scales, which approach those of logic gate operations. When transporting and separating ions on a time scale near the ions' local well oscillation periods, we observed coherent excitation of the motion that could be avoided by shaping the waveform appropriately or nearly eliminated by a coherent displacement that was applied after transport or separation. In addition, multi-ion crystals were reliably partitioned into two groups of predetermined numbers. These methods can reduce the time overhead for separation, transport, and recooling in scalable implementations of large quantum information algorithms.

The spatial extent of the separating wedge is governed by the ion-to-electrode distances; therefore, smaller traps can accomplish fast separation while maintaining stronger confinement, which would lead to reduced excitation if background motional heating can be suppressed. The constant velocity waveforms used here limit the transport duration to one oscillation period. In principle, it should be possible to transport like ions between two zones in arbitrarily short durations [20]. However, experiments that use a different species for sympathetic laser cooling [5-7] will require special consideration since all modes will be excited during both diabatic transport and separation.
This work is supported by IARPA, NSA, ONR, DARPA, and the NIST Quantum Information Program. We thank J. Heidecker for electronics support and D. Slichter and A. Wilson for helpful comments on the manuscript. This paper is a contribution of NIST and not subject to U.S. copyright.

*ryan.bowler@nist.gov

${ }^{\dagger}$ Current address: Department of Physics, Amherst College, Amherst, MA 01002-5000 USA.

${ }^{\ddagger}$ Current address: Institute for Quantum Electronics, ETH Zurich, 8093 Zurich, CH.

[1] J. I. Cirac and P. Zoller, Phys. Rev. Lett. 74, 4091 (1995).

[2] D. J. Wineland, C. Monroe, W. M. Itano, D. Leibfried, B. E. King, and D. M. Meekhof, J. Res. Natl. Inst. Stand. Technol. 103, 259 (1998).

[3] D. Kielpinski, C. Monroe, and D. Wineland, Nature (London) 417, 709 (2002).

[4] M. A. Rowe et al., Quantum Inf. Comput. 2, 257 (2002).

[5] J. D. Jost, J. P. Home, J. M. Amini, D. Hanneke, R. Ozeri, C. Langer, J. J. Bollinger, D. Leibfried, and D. J. Wineland, Nature (London) 459, 683 (2009).

[6] J.P. Home, D. Hanneke, J. D. Jost, J. M. Amini, D. Leibfried, and D. J. Wineland, Science 325, 1227 (2009).

[7] D. Hanneke, J.P. Home, J. D. Jost, J. M. Amini, D. Leibfried, and D. J. Wineland, Nature Phys. 6, 13 (2010).

[8] R. B. Blakestad, C. Ospelkaus, A. P. VanDevender, J. H. Wesenberg, M. J. Biercuk, D. Leibfried, and D. J. Wineland, Phys. Rev. A 84, 032314 (2011).

[9] M. D. Barrett et al., Nature (London) 429, 737 (2004).

[10] G. Huber, T. Deuschle, W. Schnitzler, R. Reichle, K. Singer, and F. Schmidt-Kaler, New J. Phys. 10, 013004 (2008).

[11] A. Couvert, T. Kawalec, G. Reinaudi, and D. GueryOdelin, Europhys. Lett. 83, 13001 (2008).

[12] D. M. Meekhof, C. Monroe, B. E. King, W. M. Itano, and D. J. Wineland, Phys. Rev. Lett. 76, 1796 (1996); Phys. Rev. Lett. 77, 2346(E) (1996).

[13] R. Reichle, D. Leibfried, R. B. Blakestad, J. Britton, J. D. Jost, E. Knill, C. Langer, R. Ozeri, S. Seidelin, and D. J. Wineland, Fortschr. Phys. 54, 666 (2006).

[14] S. Schulz, U. Poschinger, K. Singer, and F. Schmidt-Kaler, Fortschr. Phys. 54, 648 (2006).

[15] E. Torrontegui, S. Ibáñez, X. Chen, A. Ruschhaupt, D. Guéry-Odelin, and J. G. Muga, Phys. Rev. A 83, 013415 (2011).

[16] H.-K. Lau and D. F. V. James, Phys. Rev. A 83, 062330 (2011).

[17] D. Leibfried, R. Blatt, C. Monroe, and D. Wineland, Rev. Mod. Phys. 75, 281 (2003).

[18] J. P. Home and A. M. Steane, Quantum Inf. Comput. 6, 289 (2006).

[19] X. Chen, A. Ruschhaupt, S. Schmidt, A. del Campo, D. Guéry-Odelin, and J.G. Muga, Phys. Rev. Lett. 104, 063002 (2010).

[20] D. J. Wineland, Int. School of Physics Enrico Fermi, edited by F. De Martini and C. Monroe (IOS Press, Amsterdam, 2002), Vol. 148, p. 165.

[21] J. Jost, Ph.D. thesis, University of Colorado Boulder, 2010. 